\begin{tabular}{|c|l|}
\hline Title & $\begin{array}{l}\text { Fabrication of one dimensional GaA s channel-coupled InA s quantum dot memory device by selective area metal- } \\
\text { organic vapor phase epitaxy }\end{array}$ \\
\hline Author(s) & Nataraj, Devaraj; Ooike, Noboru; Motohisa, Junichi; Fukui, Takashi \\
\hline Citation & $\begin{array}{l}\text { A pplied Physics Letters, 87, 19310 } \\
\text { https://doi.org/10.1063/2120905 }\end{array}$ \\
\hline Issue Date & 2005-11-07 \\
\hline Doc URL & http://hdl.handle.net/2115/5504 \\
\hline Rights & Copyright $\odot$ 2005 A merican Institute of Physics \\
\hline Type & article \\
\hline File Information & APL 87-19.pdf \\
\hline
\end{tabular}

Instructions for use 


\title{
Fabrication of one-dimensional GaAs channel-coupled InAs quantum dot memory device by selective-area metal-organic vapor phase epitaxy
}

\author{
Devaraj Nataraj, ${ }^{\text {a) }}$ Noboru Ooike, Junichi Motohisa, and Takashi Fukui \\ Research Center for Integrated Quantum Electronics, Hokkaido University, North 13 West 8, \\ Sapporo 060-8628, Japan
}

(Received 12 May 2005; accepted 1 September 2005; published online 1 November 2005)

\begin{abstract}
Narrow wirelike openings were defined on $\mathrm{SiO}_{2}$-masked $\mathrm{GaAs}$ (001) substrates by electron-beam lithography and wet chemical etching methods. A one-dimensional GaAs channel-coupled InAs quantum dot memory device was fabricated in this opened area by the selective-area metal-organic vapor phase epitaxy. Drain current measurement by sweeping the gate voltage forward and backward showed clear hysteresis up to $180 \mathrm{~K}$ due to electrons charging into the quantum dots with a threshold voltage difference $\left(\Delta V_{\mathrm{th}}\right)$ of $165 \mathrm{mV}$ at $20 \mathrm{~K}$ and $29 \mathrm{mV}$ at $180 \mathrm{~K}$. Comparison of experimental $\Delta V_{\text {th }}$ values with the theoretically calculated ones showed that around 300 and 50 electrons were responsible for the memory operation at 20 and $180 \mathrm{~K}$, respectively. Real time measurements showed that the write/erase states of the memory device were discriminated for more than $5 \mathrm{~min}$ at $20 \mathrm{~K}$ and about $100 \mathrm{~s}$ at $77 \mathrm{~K}$. (C) 2005 American Institute of Physics.
\end{abstract}

[DOI: $10.1063 / 1.2120905$ ]

There has been growing interest in quantum dot information storage devices using quantum dots (QDs) as storage media. The operation of a quantum dot memory (QDM) device depends on the discrete emission and capture of electrons, and not on the average behavior of hundreds of thousands of electrons, as is the case in today's flash memory devices. In general, the QDM devices mainly consist of a quantum dot layer to store electrons and a channel in close vicinity to sense them. ${ }^{1-5}$ The storing and releasing of electrons into and from the QD energy levels is solely determined by the voltage of a control gate. While many studies of silicon QDM devices have addressed various key features, such as fabrication, room-temperature operation, the single electron charging effect, etc., ${ }^{1,2}$ only a few have addressed InAs QDM devices and most of them comprising several hundreds or thousands of QDs. ${ }^{3-5}$

Since storing information in an economic way requires high packaging density and low power consumption, the number of QDs must be reduced, and, consequently, the sensing channel should also be sufficiently reduced to enable sensing of a small potential change in the QDs caused by charging and discharging effects. Selective-area metalorganic vapor phase epitaxy (SA-MOVPE) growth is a key technology in fabricating various kinds of semiconductor nanostructures and their integrated circuits in high density. ${ }^{6}$ We have, therefore, attempted to fabricate an InAs QDM device using SA-MOVPE. The self-limited growth mechanism associated with SA-MOVPE grown GaAs wires on masked GaAs (001) substrates naturally reduces the top width. ${ }^{6,7}$ We utilized this natural width reduction mechanism to define narrow channels and then grew a few InAs QDs on the top to complete a memory structure requiring few electrons to operate.

We first defined a narrow wirelike opening with a length of $4 \mu \mathrm{m}$ in the [-110] direction and a width of $600 \mathrm{~nm}$ in the [110] direction on $\mathrm{SiO}_{2}$-masked GaAs (001) substrates by electron-beam lithography and wet chemical etching. Using

${ }^{a)}$ Electronic mails: d.nataraj@1ycos.com,nataraj@rciqe.hokudai.ac.jp a low-pressure MOVPE system operating at 76 Torr, we grew a GaAs double-hetero-high-electron mobility transistor (DH-HEMT) channel $^{6}$ in this opened area, followed by InAs QDs to complete the memory structure. The growth sequence of the channel and the related thicknesses were a $250 \mathrm{~nm}$ GaAs buffer layer, a $50 \mathrm{~nm} \mathrm{Al}_{0.3} \mathrm{Ga}_{0.7} \mathrm{As}$ layer, a $18 \mathrm{~nm}$ GaAs well layer, a $10 \mathrm{~nm} \mathrm{Al}_{0.3} \mathrm{Ga}_{0.7} \mathrm{As}$ spacer layer, a $25 \mathrm{~nm}$ Si-doped $\mathrm{Al}_{0.3} \mathrm{Ga}_{0.7} \mathrm{As}$ layer, and a $10 \mathrm{~nm}$ GaAs capping layer. The growth temperature was $700{ }^{\circ} \mathrm{C}$. After fabrication of the channel, the growth temperature was reduced to $440{ }^{\circ} \mathrm{C}$ in $5 \mathrm{~min}$, and then InAs QDs were grown in Stranski-Krastanow growth mode. A 20-nm-thick GaAs capping layer was grown at the same temperature without interruption to cover the dots, then the temperature was increased to $600{ }^{\circ} \mathrm{C}$ for a further growth of 50 -nm-thick GaAs as a second capping layer. Two similar structures were also grown for comparative and quantum dot analysis, in which one sample had no QDs layer and the other had no top capping layer. The source materials used for this growth were trimethylgallium (TMG), trimethylaluminum (TMA), trimethylindium (TMI), and 20\% arsine in purified hydrogen. The partial pressures of the TMG and TMA were 3.4 $\times 10^{-6}$ and $6.8 \times 10^{-7} \mathrm{~atm}$, respectively. The partial pressure of the arsine was $1.1 \times 10^{-4}$ atm for the GaAs well layer and $5.3 \times 10^{-4}$ atm for the AlGaAs layer. The partial pressures of the TMI and arsine for the InAs QD layer were $4.2 \times 10^{-7}$ and $5.3 \times 10^{-4} \mathrm{~atm}$, respectively. After the completion of growth, $\mathrm{Ge} / \mathrm{Au} / \mathrm{Ni} / \mathrm{Au}$ metals were deposited and annealed at $450{ }^{\circ} \mathrm{C}$ for $5 \mathrm{~min}$ as an Ohmic contact, and then $\mathrm{Cr} / \mathrm{Au}$ metals were deposited as gate electrodes. Electron-beam lithography and photolithography, together with the standard lift-off process, were used to define the electrodes. The carrier density and mobility of two-dimensional electron gas (2DEG) on a planar reference sample at $77 \mathrm{~K}$ were $1.0 \times 10^{12} \mathrm{~cm}^{-2}$ and $53700 \mathrm{~cm}^{2} / \mathrm{V} \mathrm{s}$, respectively.

Figures 1(a) and 1(b) show a scanning electron microscope (SEM) image of the SA-MOVPE grown device structure and a schematic representation of the memory device and its equivalent circuit. Because of the self-limited growth 


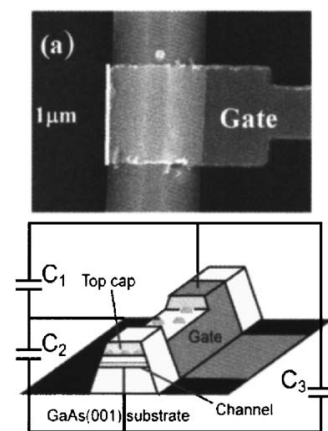

(b)

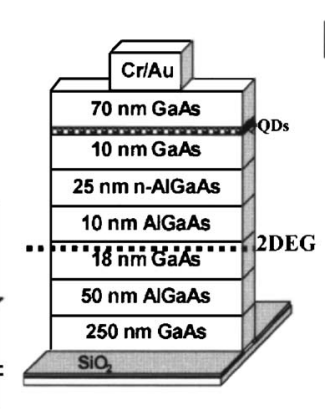

(c)

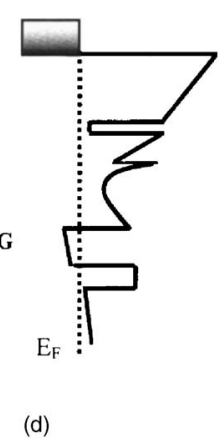

(d)

FIG. 1. (a) SEM image of SA-MOVPE grown memory structure, (b) equivalent circuit, (c) growth sequence, and (d) schematic conduction-band profile.

mechanism, the top width of the wire was reduced to $200 \mathrm{~nm}$, as shown in Fig. 1(a). Figures 1(c) and 1(d) show the growth layer details and the corresponding schematic conduction-band profile. To determine the number of buried QDs and the size distribution, we conducted the SEM and atomic force microscope (AFM) analyses on uncapped samples, grown separately but under similar growth conditions. The SEM analysis on an uncapped wire pattern showed InAs QDs with a size distribution of 10-30 nm and with an average density of 25 dots $/ 0.1 \mu \mathrm{m}^{2}$. Ex situ AFM investigations on uncapped planar samples showed that the QDs had a height of less than or equal to $8 \mathrm{~nm}$. The mechanism of $\operatorname{In}(\mathrm{Ga})$ As dot formation on GaAs (001) substrate is reported elsewhere. ${ }^{8}$ Details of the InAs dot formation on top of the GaAs wires by SA-MOVPE are reported elsewhere.'

The memory characteristics of our device, mounted on a cryohead where the temperature could be varied from 20 to $300 \mathrm{~K}$, were measured using a semiconductor parameter analyzer. Figure 2(a) shows the measured hysteresis behavior of our device at $20 \mathrm{~K}$. The drain current was measured, in the dark, for a fixed source drain voltage $\left(V_{\mathrm{DS}}\right)$ of $100 \mathrm{mV}$ by scanning the gate voltage $\left(V_{G}\right)$ from -0.4 to $1.0 \mathrm{~V}$ and back. The reverse scanning resulted in a positive voltage shift in the drain current, showing a clockwise hysteresis with a $\Delta V_{\text {th }}$ of $165 \mathrm{mV}$. A similar measurement on a sample with no QDs showed no hysteresis effect. Therefore, the positive shift in our sample with QDs can be attributed to electron capturing at QD energy levels.

The scanning in our sample was started from $-0.4 \mathrm{~V}$, in steps of $0.1 \mathrm{~V}$, up to a positive maximum gate voltage $V_{G_{\max }}$,
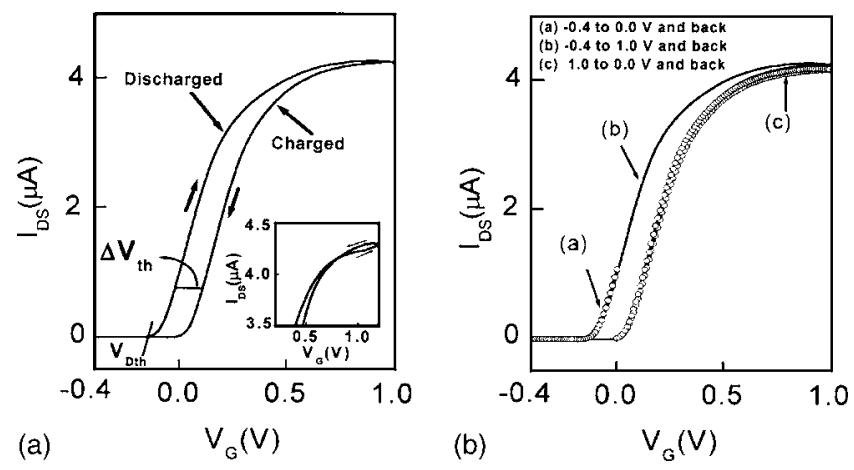

FIG. 2. (a) Hysteresis at $20 \mathrm{~K}$. Insert at right bottom is reverse loop obtained with scanning range up to $1.2 \mathrm{~V}$. (b) Hysteresis effect at three different scanning ranges.

Downloaded 23 Jan 2006 to 133.87 .26 .100 . Redistribution subject
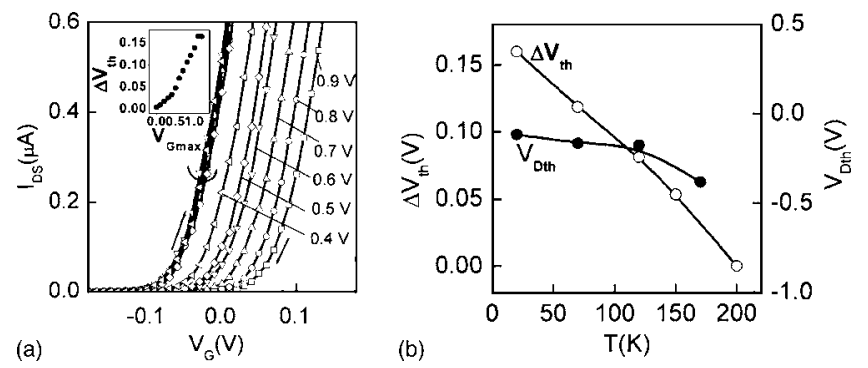

FIG. 3. (a) Hysteresis for different scanning ranges in steps of $0.1 \mathrm{~V}$, showing a clear shift in $\Delta V_{\text {th }}$ at $20 \mathrm{~K}$. Insert shows $\Delta V_{\text {th }}$ vs $V_{G_{\max }}$. (b) Temperature dependence of $\Delta V_{\text {th }}$ and $V_{D_{\text {th }}}$.

at which the QDs would be fully charged. Figure 3(a) shows that there was a clear shift in $\Delta V_{\text {th }}$, depending on the gate voltage scanning range. As the number of electrons involved in the charging process increased with the gate voltage, an increasing shift in $\Delta V_{\text {th }}$ was obtained. For the present device, $\Delta V_{\text {th }}$ increased linearly (the linear nature was due to the size distribution of the QDs) up to $1.0 \mathrm{~V}$ and then saturated, as shown in the insert of Fig. 3(a). This means that scanning between -0.4 and $1.0 \mathrm{~V}$ and back corresponds to a fully charged state.

The charging and discharging process in our device works as follows. In the forward scanning, the QD energy levels are lowered below the Fermi level, so electrons are transferred from the channel to the QD energy levels, leading to a decrease in the channel carrier concentration. During the reverse scanning, because the stored electrons are not released immediately, the magnitude of the current flowing through the channel is less, resulting in clockwise hysteresis. However, when the reverse scanning reaches $-0.4 \mathrm{~V}$, the QD energy levels are shifted above the Fermi level, so the stored electrons are completely transferred to the channel. The charging and discharging operation of our device is thus completely electrically controllable and does not required any light illumination, which is in contrast with the recent experiment by Koike et al. ${ }^{3}$ in which light illumination was required for discharge because of thick ${ }^{5}$ high barrier material between the channel and the InAs QD layer. When we increased the gate voltage scan range up to $1.2 \mathrm{~V}$, a reverse loop was obtained, as shown in the inset of Fig. 2(a) and it was similar to the one observed by Yusa and Sakaki. ${ }^{5}$ The magnitude of the reverse current within the reverse loop was higher than the forward current, and the reason for this higher magnitude could be due to the charging and immediate discharging of electrons from the excited states, probably from the $d$ levels of the InAs QDs.

We investigated the temperature dependency of our device by scanning the gate voltage forth and back at different temperatures. Figure 3(b) shows the temperature dependence on $\Delta V_{\text {th. }}$. There was a downward trend from an initial $\Delta V_{\text {th }}$ of $165 \mathrm{mV}$ at $20 \mathrm{~K}$ to zero at $200 \mathrm{~K}$. Thermal-energy-assisted excitation and removal of trapped electrons ${ }^{10}$ are the reasons for this downward trend. Recently, Balacco et al. ${ }^{11}$ have noticed room-temperature memory operation in a similar device and attributed the same to deep levels associated with InAs QDs. In our case, absence of such high-temperature operation is an indication that there are no deep levels in our device. Similar kind of InAs QD devices, free from deep levels have been prepared and reported elsewhere. ${ }^{12,13}$ Figure 3(b) also shows the position of the discharged state $\left(V_{D_{t}}\right)$ 

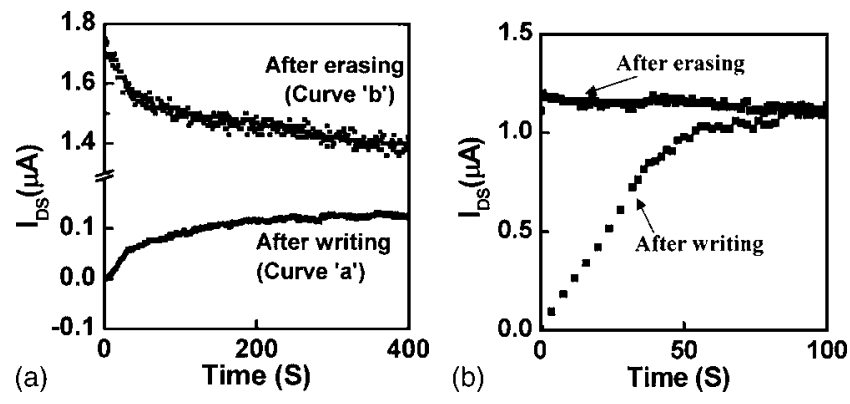

FIG. 4. Real time measurements at (a) 20 and (b) $77 \mathrm{~K}$.

curve as a function of temperature. It was slightly shifted towards the negative gate voltage, meaning that the whole hysteresis curve was shifted towards the negative gate voltage.

We also attempted to determine the nature of the charged and erased states with respect to time. We first charged QDs with electrons by applying a positive gate voltage of $1.2 \mathrm{~V}$. We then measured the drain current, in real time, at a reading voltage of $V_{G}=0.0 \mathrm{~V}$ with $V_{\mathrm{DS}}=100 \mathrm{mV}$. Previously, to determine the reading voltage, the device was scanned at two different scanning ranges "a" and "c," as shown in Fig. 2(b). As scans a (-0.4-0.0 V and back) and c (1.0-0.0 V and back) followed, respectively, the discharged and charged states curve without much deviation, the reading voltage of our device was taken to be $V_{G}=0 \mathrm{~V}$.

Figure 4(a) shows the results of real time measurement at $20 \mathrm{~K}$. Curve a is the measured drain current after charging. A fraction of the stored electrons returned to the channel, giving rise to an upward trend in the drain current, because some of the occupied dot levels had higher energies than the Fermi level. Curve $b$ is the measured drain current after discharging. The downward trend was due to the loss in the channel electron concentration, followed by a transfer of electrons to the QDs by tunneling.

Furthermore, from Fig. 4(a), we can see that the write and erase states of our memory device could be discriminated for more than $5 \mathrm{~min}$. at $20 \mathrm{~K}$. However, a similar measurement at $77 \mathrm{~K}$ showed that they could be differentiated for no more than $100 \mathrm{~s}$, as shown in Fig. 4(b). This was due to thermal-energy-assisted excitation and emission across the barrier, in either direction, from dot to channel or channel to dot, respectively, after writing or erasing. The results of our real time measurements, however, are not sufficient for us to determine the retention characteristics ${ }^{3}$ of our memory device. We plan to investigate them more completely.

Finally, we calculated the $\Delta V_{\text {th }}\left(\Delta V_{\text {th }}=n q / C_{\text {Total }}\right)$ of our device by taking into account the total capacitance $\left(C_{\text {Total }}\right)$ of its structure, where $n$ is number of electrons stored in the QD energy levels and $q$ is the electric charge. From Fig. 1(b), we obtained $^{1}$ the total capacitance of our device structure as $C_{\text {Total }}=C_{1}+\left[\left(C_{1}+C_{2}\right) / C_{2}\right] C_{3}$, where $C_{1}$ is the capacitance between the gate and the dot, $C_{2}$ is the capacitance between the dot and the channel, and $C_{3}$ is the capacitance between the gate and the channel. If we define $C_{1}=\varepsilon_{r} A_{d} / d_{1}, C_{2}$ $=\varepsilon_{r} A_{d} / d_{2}$, and $C_{3}=\varepsilon_{r}\left(A-A_{d}\right) /\left(d_{1}+d_{2}\right)$ and assume that the dielectric constants are equal for $\mathrm{GaAs}$ and $\mathrm{Al}_{0.3} \mathrm{Ga}_{0.7} \mathrm{As}$, we can rewrite $C_{\text {Total }}$ as $C_{\text {Total }}=A \varepsilon_{r} / d_{1}$. This means that $\Delta V_{\text {th }}$ $=n q d_{1} / A \varepsilon_{r}$, where $A_{d}$ is the InAs dot area, $A$ is the area of the channel under the gate, $d_{1}$ is the distance from the gate to the dot, and $d_{2}$ is the distance from the dot to the channel. Therefore, to estimate the $\Delta V_{\text {th }}$ of our device, the number of dots buried under the gate (50 dots as observed from SEM analysis of uncapped wire samples), the channel dimension and top capping thickness $(70 \mathrm{~nm})$ should be known. In the present device structure, the top wire dimension under the gate $(200 \times 1000 \mathrm{~nm})$ was considered as the channel dimension. Further, assuming that the number of electrons in each QD is equal to six, two $s$ and four $p$ shell electrons of an InAs QD, we calculate the $\Delta V_{\text {th }}$ to be $147 \mathrm{mV}$ (occupation of six electrons per InAs QD has been reported elsewhere ${ }^{14}$ ). The calculated value is comparable to the experimentally obtained value of $165 \mathrm{mV}$ at $20 \mathrm{~K}$, indicating that around 300 electrons were responsible for memory operation at this temperature. A similar calculation at $180 \mathrm{~K}$ resulted in a value of $24 \mathrm{mV}$, with a single electron per QD, and this was also comparable with the experimental value of $29 \mathrm{mV}$. We therefore attribute this high-temperature $\Delta V_{\text {th }}$ value to the single electron charging effect.

In conclusion, we have successfully fabricated a onedimensional GaAs channel-coupled InAs quantum dot memory device by SA-MOVPE and demonstrated the existence of memory up to $180 \mathrm{~K}$. We used a natural width reduction mechanism associated with the self-limited growth mechanism of SA-MOVPE to define narrow channels and then grew a few InAs QDs on the top of the channel to realize a memory device involving few electrons (around 300 and 50 at 20 and $180 \mathrm{~K}$, respectively). Further investigation is now underway to fabricate an InAs quantum dot memory device with even more reduced dimensions to realize single dot single electron memory that can operate at room temperature.

One of the authors (D.N.) would like to thank Professor Hideki Hasegawa and Professor Yuzuru Tanaka for their encouragement and support during this project.

${ }^{1}$ L. Guo, E. Leobandung, L. Shuang, and S. Y. Chou, J. Vac. Sci. Technol. B 15, 2840 (1997).

${ }^{2}$ W. Wu, J. Gu, H. Ge, C. Keimal, and S. Y. Chou, Appl. Phys. Lett. 83, 2268 (2003).

${ }^{3}$ K. Koike, K. Saitoh, S. Li, S. Sasa, M. Inoue, and M. Yano, Appl. Phys. Lett. 76, 1464 (2000).

${ }^{4}$ G. Yusa and H. Sakaki, Superlattices Microstruct. 25, 247 (1999).

${ }^{5}$ G. Yusa and H. Sakaki, J. Cryst. Growth 175/176, 730 (1997).

${ }^{6}$ F. Nakajima, Y. Miyoshi, J. Motohisa, and T. Fukui, Appl. Phys. Lett. 83, 2680 (2003).

${ }^{7}$ K. Kumakura, J. Motohisa, and T. Fukui, Jpn. J. Appl. Phys., Part 1 34, 4387 (1995).

${ }^{8}$ P. R. Berger, K. Chang, P. Bhattacharya, J. Singh, and K. K. Bajaj, Appl. Phys. Lett. 53, 684 (1988).

${ }^{9}$ C. K. Hahn, J. Motohisa, and T. Fukui, Appl. Phys. Lett. 76, 3947 (2001).

${ }^{10}$ W.-H. Chang, W. Y. Chen, M. C. Cheng, C. Y. Lai, and T. M. Hsu, Phys. Rev. B 64, 125315 (2001).

${ }^{11}$ C. Balocco, A. M. Song, and M. Missous, Appl. Phys. Lett. 85, 5911 (2004).

${ }^{12}$ V. Davydov, I. Ignatiev, H.-W. Ren, S. Sugou, and Y. Masumoto, Appl. Phys. Lett. 74, 3002 (1999).

${ }^{13}$ C. M. A. Kapteyn, F. Heinrichsdorff, O. Stier, R. Heitz, M. Grundmann, N. D. Zakharov, D. Bimberg, and P. Werner, Phys. Rev. B 60, 14265 (1999).

${ }^{14}$ K. Yoh, J. Konda, S. Shina, and N. Nishiguchi, Jpn. J. Appl. Phys., Part 1 36, 4134 (1997). 